\title{
Patient and health system delays before registration among migrant patients with tuberculosis who were transferred out in China
}

Tao Li ${ }^{1}$, Hui Zhang ${ }^{1}$, Hemant Deepak Shewade ${ }^{2}$, Kyaw Thu Soe ${ }^{3}$, Lixia Wang ${ }^{1}$ and Xin Du*

\begin{abstract}
Background: Early diagnosis and treatment is vital for effective tuberculosis (TB) management especially among migrant populations who are a vulnerable group. We aimed to study factors associated with delay before registration at country level among registered migrant TB patients in China (2014-15) who were transferred out (during treatment) through web-based TB information management system (TBIMS).

Methods: This was a cross sectional study involving review of TBIMS data. Delays (in days) were classified as follows: patient delay (from symptom onset to first doctor visit), health system delay (from first doctor visit to treatment initiation, divided into health system diagnosis and treatment delay before and after date of diagnosis respectively), diagnosis delay (from symptom onset to diagnosis) and total delay (from symptom onset to treatment initiation). Linear regression was used to build a predictive model (forward stepwise) for the socio-demographic, clinical and health system related factors associated with delay: one model for each type of delay. Delays were log transformed and included in the model.

Results: The median (IQR) patient delay, health system delay and total delay was $16(6,34)$, two $(0,6)$ and $22(11,41)$ days respectively. Factors associated with long patient, diagnosis and total delay were: female gender, age $\geq 65$ years, sputum smear positive pulmonary TB and registration at referral hospital. Treatment initiation delay was significantly higher among those registered in referral hospitals, unemployed and previously treated. Among migrant patients having permanent residence out of province, health system diagnosis delay was significantly higher while treatment initiation delay after diagnosis was significantly lower when compared to patients having permanent residence within the prefecture.

Conclusion: Among migrant population with TB, patient delay contributed to the total delay. The factors identified including the need for improved coordination between referral hospitals and national programme have to be addressed if China has to end TB.
\end{abstract}

Keywords: Patient delay, Health system delay, Diagnosis delay, Migrant, Transferred out, China, Operational research

\footnotetext{
* Correspondence: duxin@chinacdc.cn

${ }^{1}$ National Center for Tuberculosis Control and Prevention, China CDC, No.

155 Changbai Road, Changping District, Beijing 102206, China

Full list of author information is available at the end of the article
}

(c) The Author(s). 2018 Open Access This article is distributed under the terms of the Creative Commons Attribution 4.0 International License (http://creativecommons.org/licenses/by/4.0/), which permits unrestricted use, distribution, and reproduction in any medium, provided you give appropriate credit to the original author(s) and the source, provide a link to the Creative Commons license, and indicate if changes were made. The Creative Commons Public Domain Dedication waiver (http://creativecommons.org/publicdomain/zero/1.0/) applies to the data made available in this article, unless otherwise stated. 


\section{Background}

Globally, tuberculosis (TB) is the leading cause of death from infectious diseases. There were an estimated 10.4 million new TB patients in 2016, and 1.7 million died from the disease [1]. Early diagnosis and treatment is vital for effective TB management and is emphasized in World Health Organization (WHO) 'The End TB strategy' [2]. Delays in seeking diagnosis and treatment can result in severe clinical presentation at treatment initiation and unfavourable outcomes [3, 4].

China is listed as one of the 30 TB high burden countries, and has the third largest number of cases all over the world, with an estimated 895,000 (766000-1,030,000) incident cases in 2016 [1]: Migrant TB populations are always considered as important vulnerable groups in TB control [5]. In China, migrant populations were mostly internal migrants than cross-border immigrants. Internal migrants (henceforth called as migrants) account for one-fifth of the whole population [6, 7]. In 2010, 29,924 new migrant patients with smear positive pulmonary TB (PTB) were registered, accounting for $7 \%$ of total new smear positive cases nationwide [8].

Globally, time delays and factors associated with delays among TB patients (among migrants or local residents) has been studied extensively [9-13]. Migrants have many specific characteristics such as instability, low income, poor living, and working condition which could obviously influence on their health seeking behavior and cause longer delay than general population $[5,14-18]$. The inequality of social insurance coverage between local residents and migrant populations can further aggravate the delays [19-21].

Programmatically, two possible reasons for poor TB treatment outcomes among migrants are i) patient and health system delays before registration for treatment ii) high transfer outs during treatment which may contribute to treatment non evaluation. To address non-evaluation among transfer-outs, China has implemented web-based transfer-out using TB information management system (TBIMS) [22].

There have been many studies from China (among migrants and non-migrants) on delays in $\mathrm{TB}$ diagnosis and treatment [11, 12, 23-25]. Few focused on migrant population, none of them interpreted all stages of delays systematically and none were based on national level data. Furthermore, there was no study specifically addressing transferred-out migrant patients with TB. This subgroup of patient among migrant TB has higher risk of unfavourable outcomes. At the time of patient registration, the TBIMS systematically collects information on symptom onset and first doctor visit along with date of diagnosis and treatment initiation. It also collects information on permanent residence. This provides us an opportunity to study risk factors for delay at country level among migrant TB patients that were transferred out during treatment in China. The findings on extent of treatment non evaluation among transferred out migrant TB patients and its risk factors will be published elsewhere.

\section{Methods \\ Study design}

This is a cross sectional study involving review of TBIMS data.

\section{Setting \\ General setting}

China is the world's most populous country with a population of over 1.4 billion [1]. The sub-national administrative division consists of province or regions $(n=34)$, prefectures $(n=333)$ and counties $(>3000)$.

The National center for tuberculosis control and prevention (NCTB) belongs to Chinese center for disease control and prevention and is in charge of National TB programme. TB management units are established at provincial, prefecture and county levels (basic management units (BMU) at county level), including independent TB dispensaries, TB control divisions within local centers for disease control and prevention and health facilities designated by health authorities. TB diagnostic facilities are centralized at the county level and rarely also available at township level (below county). At BMUs, diagnosed patients are registered and initiated on directly observed therapy-short course (DOTS). They are assisted by township clinics and village health workers. Anti-tuberculosis drugs and needful examinations are provided without charge. Similar to a BMU, some regional referral hospitals also take patient management responsibility and register patients. Unlike in BMUs, the drugs and examinations are usually not free in referral hospitals. The payment could be reimbursed by social insurances but migrant population often have many barriers to get reimbursed [26].

\section{China web-based TBIMS}

Patients are registered for treatment in the web-based TBIMS. In January 2005, the Ministry of Health of China launched the first version of TBIMS. The second version of TBIMS was launched in April 2009. The transfer-in/out module was added in this update. The function of TBIMS is divided into 4 groups: data collection, quality assessment, and output and system management [27] (Fig. 1).

TBIMS routinely collects individual patients' information as well as national TB programme (NTP) activities. Patients' standard medical records are filled by doctors and entered into TBIMS by NTP health care workers, including all diagnosis, treatment, follow-up examinations, 


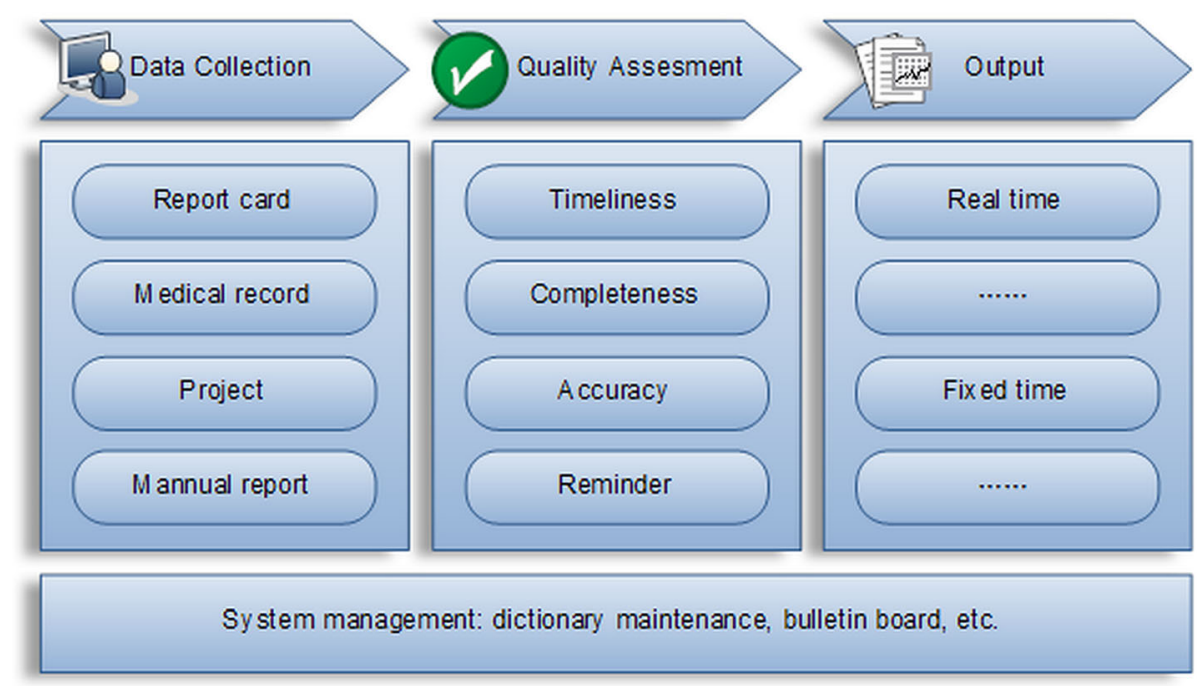

Fig. 1 Diagram depicting functions of electronic TB information management system (TBIMS), China

outcome and DOTS management information. NTP activities that are updated in TBIMS include training, supervision, meeting and health education. All data are restored in a centralized national data center run by China center for disease control. Individual cases and statistical reports can be generated, viewed, edited and extracted for patients care and programme management.

The transfer of patients with TB is implemented by BMU through TBIMS and the transfer-in BMU will be noticed in the system to trace the patient. If the patients were traced successfully, the transfer-in BMU will take over patients' management, and update the outcome in TBIMS when patients finished their treatment.

\section{Study population and period}

All migrant patients with TB registered in China during 2014-15 and transferred-out using web-based TBIMS anytime during their treatment were the study population.

Migrant patient with TB refers to those who were diagnosed as $\mathrm{TB}$ and registered at county $\mathrm{BMU} /$ referral hospital, were from another BMU, and stayed for less than 6 months within that county at the time of registration. Migrant status at registration is routinely captured in the TBIMS.

\section{Data collection}

Secondary data was extracted from electronic TBIMS in Microsoft Excel (Microsoft, Redmond, WA, USA). Sociodemographic (age, gender, occupation and permanent residence of patient), clinical (TB classification, treatment category and HIV status) variables and BMU name were collected at the time of registration. In addition the following dates were also collected: symptom onset, first doctor visit, diagnosis, registration, treatment initiation.

\section{Data management and analysis}

Database was constructed, cleaned and analyzed with Microsoft Excel (Microsoft, Redmond, WA, USA). Adjusted analysis was done using STATA (version 12.1, copyright 1985-2011 Stata Corp LP USA).

\section{Derived variables}

'Whether registered at referral hospital' was derived based on BMU name. Delays were calculated from different dates: patient delay (from symptom onset to first doctor visit), health system delay (from first doctor visit to treatment initiation, divided into health system diagnosis delay and treatment delay before and after date of diagnosis respectively), diagnosis delay (from symptom onset to diagnosis) and total delay (from symptom onset to treatment initiation) (Fig. 2).

\section{Data analysis}

Median and interquartile range (IQR) were used to summarize delays. Linear regression was used to build a predictive model (forward stepwise) for factors associated with delay: one model for each type of delay. Log transformation of delay variable (as it was not normally distributed) was done and included as outcome variable in each model. Age, gender and variables with unadjusted $p$ value $<0.20$ were considered in the models. First, the variable with lowest unadjusted $\mathrm{p}$ value was added followed by the variable with the next lowest unadjusted $\mathrm{p}$ value. At each step, the variable was retained if the likelihood ratio test was significant $(p<0.05)$. Beta coefficients $(0.95 \mathrm{CI})$ were 


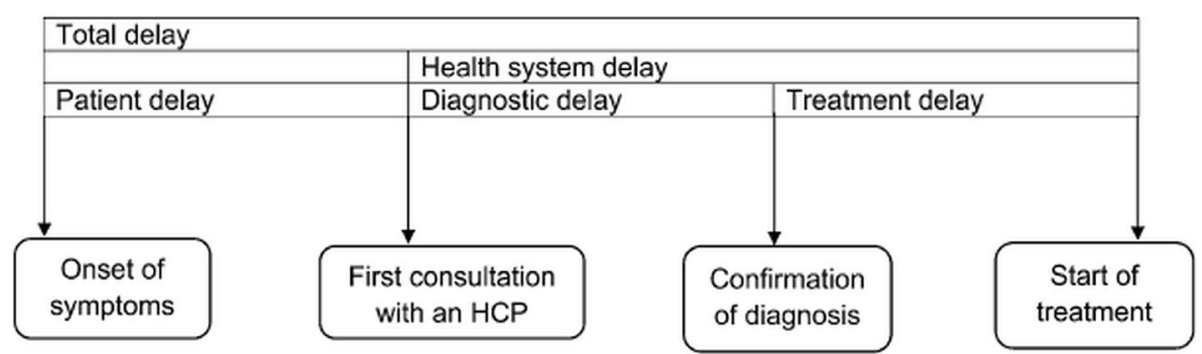

Fig. 2 Conceptual framework on definitions of delay before treatment initiation among migrant TB patients that were transfer out using web-based TBIMS in China (2014-15)* [29]. *Reprinted with permission of the International Union Against Tuberculosis and Lung Disease. Copyright $\odot$ The Union [29]. HCP - Health Care Providers

used to summarize (infer) the association of the variables included in the final model with delay.

\section{Results}

There were 7284 patients. Various delays stratified by socio-demographic, clinical and health system level characteristics have been summarized in Tables 1 and 2 .
Because of missing dates, a total of 22 patient records were excluded and 7262 records were including in building the models.

\section{Patient delay}

The median (IQR) patient delay was $16(6,34)$ days. Final model for independent predictors for patient

Table 1 Patient and health system delay before registration among transferred out migrant patients with TB $^{\mathrm{a}}$, stratified by their socio-demographic characteristics, China, 2014-2015

\begin{tabular}{|c|c|c|c|c|c|c|c|}
\hline \multirow[t]{2}{*}{ Characteristics } & $\begin{array}{l}\text { Total patients } \\
(n=7284)\end{array}$ & $\begin{array}{l}\text { Patient delay } \\
(n=7275)\end{array}$ & $\begin{array}{l}\text { Health system } \\
\text { diagnosis delay } \\
(n=7283)\end{array}$ & $\begin{array}{l}\text { Health system } \\
\text { treatment delay } \\
(n=7270)\end{array}$ & $\begin{array}{l}\text { Diagnosis delay } \\
(n=7269)\end{array}$ & $\begin{array}{l}\text { Health system } \\
\text { delay }(n=7275)\end{array}$ & $\begin{array}{l}\text { Total delay } \\
(n=7262)\end{array}$ \\
\hline & N (\%) & Median (IQR) & Median (IQR) & Median (IQR) & Median (IQR) & Median (IQR) & Median (IQR) \\
\hline Total & $7284(100)$ & $16(6,34)$ & $1(0,5)$ & $0(0,0)$ & $21(10,40)$ & $2(0,6)$ & $22(11,41)$ \\
\hline \multicolumn{8}{|l|}{ Age group (in years) } \\
\hline$<15$ & $33(0.5)$ & $14(5,30)$ & $2(0,3)$ & $0(0,1)$ & $19(10,32)$ & $2(1,3)$ & $19(10,32)$ \\
\hline $15-44$ & $4261(58.5)$ & $15(4,33)$ & $1(0,6)$ & $0(0,0)$ & $20(9,40)$ & $2(0,6)$ & $21(10,41)$ \\
\hline $45-64$ & $2126(29.2)$ & $19(7,35)$ & $2(0,5)$ & $0(0,0)$ & $23(11,42)$ & $2(0,5)$ & $23(12,43)$ \\
\hline$>=65$ & $864(11.8)$ & $18(8,34)$ & $2(0,5)$ & $0(0,0)$ & $23(12,39)$ & $2(0,5)$ & $23(12,40)$ \\
\hline \multicolumn{8}{|l|}{ Gender } \\
\hline Male & $5107(70.1)$ & $15(5,33)$ & $1(0,5)$ & $0(0,0)$ & $21(10,39)$ & $2(0,5)$ & $21(10,40)$ \\
\hline Female & $2177(29.9)$ & $17(6,37)$ & $2(0,5)$ & $0(0,0)$ & $23(11,44)$ & $2(0,6)$ & $23(12,44)$ \\
\hline \multicolumn{8}{|l|}{ Occupation } \\
\hline Studying & $492(6.8)$ & $9(2,26)$ & $2(0,8)$ & $0(0,1)$ & $17(8,31)$ & $3(1,9)$ & $18(8,32)$ \\
\hline Farmers and herdsmen & $2321(31.8)$ & $22(7,37)$ & $2(0,4)$ & $0(0,0)$ & $27(12,42)$ & $2(0,5)$ & $28(12,42)$ \\
\hline Semi-skilled employee & $107(1.5)$ & $10(1,31)$ & $3(0,11)$ & $0(0,0)$ & $21(7,42)$ & $3(0,11)$ & $21(7,42)$ \\
\hline Salaried employee & $1294(17.7)$ & $14(5,32)$ & $1(0,5)$ & $0(0,0)$ & $20(9,39)$ & $1(0,5)$ & $20(9,39)$ \\
\hline Non-salaried employee & $290(4.0)$ & $14(4,37)$ & $1(0,4)$ & $0(0,0)$ & $20(10,42)$ & $2(0,6)$ & $20(10,43)$ \\
\hline Unemployed & $2328(32.0)$ & $14(5,33)$ & $1(0,6)$ & $0(0,1)$ & $19(10,40)$ & $2(0,6)$ & $20(11,40)$ \\
\hline Others & $452(6.2)$ & $20(8,42)$ & $1(0,5)$ & $0(0,0)$ & $27(13,55)$ & $2(0,6)$ & $28(14,59)$ \\
\hline \multicolumn{8}{|l|}{ Permanent residence } \\
\hline Within prefecture & $4871(66.9)$ & $16(7,32)$ & $2(0,5)$ & $0(0,0)$ & $21(11,38)$ & $2(0,5)$ & $21(11,38)$ \\
\hline Within province & $557(7.6)$ & $19(5,53)$ & $1(0,4)$ & $0(0,0)$ & $26(10,60)$ & $1(0,5)$ & $26(10,62)$ \\
\hline Out of province & $1856(25.5)$ & $15(3,37)$ & $1(0,6)$ & $0(0,0)$ & $22(9,48)$ & $1(0,7)$ & $22(9,49)$ \\
\hline
\end{tabular}

Few dates missing for some patients, hence total assessed for each type of delay may not be 7284

$T B$ tuberculosis, PTB pulmonary tuberculosis, IQR interquartile range

${ }^{a}$ registered in web-based TB information management system 
Table 2 Patient and health system delay before registration among transferred out migrant patients with TB $^{\text {a }}$, stratified by their clinical and programmatic characteristics, China, 2014-2015

\begin{tabular}{|c|c|c|c|c|c|c|c|}
\hline \multirow[t]{2}{*}{ Characteristics } & $\begin{array}{l}\text { Total patients } \\
(n=7284)\end{array}$ & $\begin{array}{l}\text { Patient delay } \\
(n=7275)\end{array}$ & $\begin{array}{l}\text { Health system } \\
\text { diagnosis delay } \\
(n=7283)\end{array}$ & $\begin{array}{l}\text { Health system } \\
\text { treatment delay } \\
(n=7270)\end{array}$ & $\begin{array}{l}\text { Diagnosis delay } \\
(n=7269)\end{array}$ & $\begin{array}{l}\text { Health system } \\
\text { delay }(n=7275)\end{array}$ & $\begin{array}{l}\text { Total delay } \\
(n=7262)\end{array}$ \\
\hline & N (\%) & Median (IQR) & Median (IQR) & Median (IQR) & Median (IQR) & Median (IQR) & Median (IQR) \\
\hline$\overline{\text { Total }}$ & $7284(100)$ & $16(6,34)$ & $1(0,5)$ & $0(0,0)$ & $21(10,40)$ & $2(0,6)$ & $22(11,41)$ \\
\hline \multicolumn{8}{|l|}{ Classification } \\
\hline PTB smear positive & 2440 (33.5) & $20(7,49)$ & $1(0,4)$ & $0(0,0)$ & $24(11,57)$ & $2(0,5)$ & $25(11,59)$ \\
\hline PTB smear negative & $4324(59.4)$ & $15(4,32)$ & $2(0,6)$ & $0(0,0)$ & $20(10,37)$ & $2(0,6)$ & $21(10,38)$ \\
\hline PTB smear status unknown & $34(0.5)$ & $22(7,47)$ & $3(1,6)$ & $0(0,0)$ & $32(14,73)$ & $4(1,8)$ & $32(22,73)$ \\
\hline Pleurisy & $483(6.6)$ & $15(7,30)$ & $2(0,6)$ & $0(0,1)$ & $20(12,32)$ & $2(1,6)$ & $20(13,32)$ \\
\hline EPTB & $3(<0.1)$ & $25(17,74)$ & $0(0,0)$ & $0(0,1)$ & $25(17,74)$ & $0(0,1)$ & $25(18,74)$ \\
\hline \multicolumn{8}{|l|}{ Category } \\
\hline New & $6915(94.9)$ & $16(5,34)$ & $1(0,5)$ & $0(0,0)$ & $21(10,40)$ & $2(0,6)$ & $22(11,41)$ \\
\hline Retreated & $369(5.1)$ & $19(8,41)$ & $2(0,4)$ & $0(0,1)$ & $23(12,46)$ & $2(0,5)$ & $23(13,48)$ \\
\hline \multicolumn{8}{|l|}{ HIV } \\
\hline Positive & $9(0.1)$ & $31(20,57)$ & $2(0,10)$ & $0(0,0)$ & $44(29,59)$ & $2(0,11)$ & $44(29,59)$ \\
\hline Negative & 2864 (39.3) & $14(6,33)$ & $2(1,7)$ & $0(0,1)$ & $20(11,40)$ & $3(1,8)$ & $21(12,41)$ \\
\hline Unknown $^{\mathrm{b}}$ & $4411(60.6)$ & $18(6,35)$ & $1(0,4)$ & $0(0,0)$ & $22(10,40)$ & $1(0,4)$ & $23(10,41)$ \\
\hline \multicolumn{8}{|l|}{ Registered in referral hospital } \\
\hline Yes & $4153(57.0)$ & $17(7,33)$ & $2(1,5)$ & $0(0,0)$ & $22(11,38)$ & $2(1,6)$ & $23(11,39)$ \\
\hline No & $3131(43.0)$ & $15(4,36)$ & $1(0,5)$ & $0(0,0)$ & $21(9,44)$ & $1(0,6)$ & $21(10,46)$ \\
\hline
\end{tabular}

Few dates missing for some patients, hence total assessed for each type of delay may not be 7284

$T B$ tuberculosis, PTB pulmonary tuberculosis, EPTB extrapulmonary tuberculosis, HIV human immunodeficiency virus, IQR interquartile range

${ }^{\text {a }}$ registered in web-based TB information management system

${ }^{\mathrm{b}} \mathrm{TB}$ examinations were routinely carried out in all new or follow up HIV/AIDS patients nationwide while TB patients were screened with HIV tests only in selected high HIV epidemic counties

delay is shown in Table 3. Female gender $(\beta=0.11, p=$ $0.003)$, and patients with sputum smear positive pulmonary $\mathrm{TB}(\beta=0.33, p<0.001)$ were independent predictors for longer delay. Age between 15 and 44 years $(\beta=-0.23$, $p<0.001)$ and patients registered in programme BMU $(\beta=-0.20, p<0.001)$ were independent predictors for shorter delay. Though included in the final model, migrant patient permanent residence was not an independent predictor. Occupation, treatment category and HIV status were excluded by the model.

\section{Health system delay}

The median (IQR) health system delay was two $(0,6)$ days. Final model for independent predictors for health system delay is shown in Table 4 . Patients with unknown sputum smear status $(\beta=0.57, p=0.003)$ was independent predictor for longer delay, while unknown HIV status $(\beta=-0.40, p<0.001)$ was independent predictor for shorter delay. Age group, gender, occupation, permanent residence, treatment category and registration at referral hospital were excluded by model.

\section{Health system diagnosis delay}

The median (IQR) health system diagnosis delay was one $(0,5)$ day. Final model for independent predictors for health system diagnosis delay is shown in Table 5 . Independent predictors of health system diagnosis delay were similar to predictors of overall health system delay, but in addition sputum smear positive TB $(\beta=-0.16, p<0.003)$ was also independent predictor for shorter delay and permanent residence being out of province $(\beta=0.13, p<0.001)$ was independent predictor for longer delay. Age group, gender, occupation, treatment category and registration at referral hospital were excluded by the model.

\section{Health system treatment initiation delay}

The median (IQR) health system treatment initiation delay was zero $(0,0)$ day. Final model for independent predictors for health system treatment initiation delay is shown in Table 6. Independent predictors of health system treatment initiation delay were similar to predictors of overall health system delay, but in addition permanent residence being out of province $(\beta=-0.03, p<0.001)$, occupation being farmers/herdsmen $(\beta=-0.02, p=0.013)$ 
Table 3 Linear regression for independent predictors of patient delay before registration among transferred out migrant patients with $\mathrm{TB}^{\mathrm{a}}$, China, $2014-15^{\mathrm{b}}(\mathrm{N}=7262)^{\mathrm{c}}$

\begin{tabular}{llll}
\hline Variable & B coefficient & $95 \% \mathrm{Cl}$ & $P$ value \\
\hline Age group (in years) & & & \\
$\quad<15$ & -0.30 & $-0.82,0.21$ & 0.250 \\
$15-44$ & -0.23 & $-0.34,-0.12$ & $<0.001^{\wedge}$ \\
$45-64$ & 0.03 & $-0.09,0.14$ & 0.672 \\
$>=65$ & Ref & Ref & Ref \\
Gender & & & \\
Male & Ref & Ref & Ref \\
Female & 0.11 & $0.04,0.19$ & $0.003^{\wedge}$ \\
Classification & & & \\
PTB smear positive & 0.33 & $0.19,0.48$ & $<0.001^{\wedge}$ \\
PTB smear negative & -0.04 & $-0.18,0.10$ & 0.572 \\
PTB smear status unknown & 0.37 & $-0.14,0.89$ & 0.157 \\
Pleurisy & Ref & Ref & Ref \\
EPTB & 1.02 & $-0.66,2.70$ & 0.235 \\
Registered at referral hospital & & & \\
Yes & Ref & Ref & Ref \\
No & -0.20 & $-0.27,-0.13$ & $<0.001^{\wedge}$ \\
\hline
\end{tabular}

TB tuberculosis, $P T B$ pulmonary tuberculosis, EPTB extrapulmonary tuberculosis aregistered in web-based TB information management system

blogarithmic transformation of delay variable done as it was not normally distributed, model building was done by stepwise (forward method) method and the final model has been presented; occupation, permanent residence, treatment category and HIV status, though considered, were excluded by the model (likelihood ratio test)

$F$ stat $=20.8 ;$ Probability $>F=<0.001$

cof 7284, twenty two patient records were excluded because of missing information on dates to calculate delay

${ }^{\wedge}$ significant $p$-value $<0.05$

and salaried employee $(\beta=-0.03, p=0.002)$ and new TB patients $(\beta=-0.02, p=0.014)$ were independent predictors for shorter delay. Age group, gender and registration at referral hospital were excluded by the model.

\section{Diagnosis delay}

The median (IQR) diagnosis delay was $21(10,40)$ days. Final model for independent predictors for diagnosis delay is shown in Table 7. Independent predictors for diagnosis delay were similar to predictors of patient delay.

\section{Total delay}

The median (IQR) total delay was $22(11,41)$ days. Final model for independent predictors for total delay is shown in Table 8. Independent predictors for total delay were similar to predictors of patient delay.

\section{Summary of independent predictors}

The independent predictors of each type of delay have been summarized in Additional file 1: Table S1. The
Table 4 Linear regression for independent predictors of health system delay before registration among transferred out migrant patients with TBa ${ }^{\mathrm{a}}$ China, 2014-15 ${ }^{\mathrm{b}}(\mathrm{N}=7262)^{\mathrm{c}}$

\begin{tabular}{llll}
\hline Variable & B coefficient & $95 \% \mathrm{Cl}$ & $P$ value \\
\hline Classification & & & \\
PTB smear positive & -0.10 & $-0.21,0.002$ & 0.056 \\
PTB smear negative & 0.02 & $-0.08,0.13$ & 0.631 \\
PTB smear status unknown & 0.57 & $0.20,0.95$ & $0.003^{\wedge}$ \\
Pleurisy & Ref & Ref & Ref \\
EPTB & -0.82 & $-2.04,0.41$ & 0.193 \\
HIV & & & \\
Positive & -0.04 & $-0.75,0.67$ & 0.911 \\
Negative & Ref & Ref & Ref \\
Unknown & -0.40 & $-0.45,-0.35$ & $<0.001^{\wedge}$ \\
\hline
\end{tabular}

TB tuberculosis, PTB pulmonary tuberculosis, EPTB extrapulmonary tuberculosis, HIV human immunodeficiency virus

${ }^{a}$ registered in web-based TB information management system

${ }^{\mathrm{b}}$ logarithmic transformation of delay variable done as it was not normally distributed, model building was done by stepwise (forward method) method and the final model has been presented; age group, gender, occupation, permanent residence, treatment category and registration at referral hospital, though considered, were excluded by the model (likelihood ratio test) $F$ stat for model $=43.6$; Probability $>F=<0.001$

cof 7284, twenty two patient records were excluded because of missing information on dates to calculate delay

${ }^{\wedge}$ significant $p$-value $<0.05$

positive or negative sign in brackets indicates the direction of association: positive sign means the factor is an independent predictor for longer delay and negative sign means the factor is an independent predictor for shorter delay when compared to reference.

\section{Discussion}

This was the first country wide study from China looking at delay before registration among migrant TB patients. There were some key findings from this study. The median total delay was 22 days which was significantly contributed by median 16 days of patient delay, while health system delay was much shorter. Elderly age group, female gender, patients registered in referral hospitals and patients with pulmonary sputum smear positive TB were factors associated with longer patient delay, diagnosis delay and total delay. Patients registered in referral hospital or with previous treatment history had significant higher treatment initiation delay.

\section{Short patient and health system delays}

Patient delay significantly contributed to total delay: this was similar to other studies in India, China and Asia [28-30]. The median total delay (22 days) and patient delay (16 days) were quite shorter compared to studies in other high TB burden countries [28, 30]. Migrant TB patients received proper diagnosis and management within very short duration of having any symptom of $\mathrm{TB}$ 
Table 5 Linear regression for independent predictors of health system diagnosis delay before registration among transferred out migrant patients with $\mathrm{TB}^{\mathrm{a}}$, China, 2014-15 ${ }^{\mathrm{b}}(\mathrm{N}=7262)^{\mathrm{c}}$

\begin{tabular}{|c|c|c|c|}
\hline Variable & B coefficient & $95 \% \mathrm{Cl}$ & $P$ value \\
\hline \multicolumn{4}{|l|}{ Permanent residence } \\
\hline Within prefecture & Ref & Ref & Ref \\
\hline Within province & -0.004 & $-0.10,0.09$ & 0.932 \\
\hline Out of province & 0.13 & $0.07,0.19$ & $<0.001^{\wedge}$ \\
\hline \multicolumn{4}{|l|}{ Classification } \\
\hline PTB smear positive & -0.16 & $-0.27-0.06$ & $0.003^{\wedge}$ \\
\hline PTB smear negative & 0.01 & $-0.09,0.12$ & 0.784 \\
\hline PTB smear status unknown & 0.40 & $0.02,0.77$ & $0.037^{\wedge}$ \\
\hline Pleurisy & Ref & Ref & Ref \\
\hline EPTB & -0.77 & $-1.99,0.45$ & 0.218 \\
\hline \multicolumn{4}{|l|}{ HIV } \\
\hline Positive & 0.06 & $-0.64,0.77$ & 0.864 \\
\hline Negative & Ref & Ref & Ref \\
\hline Unknown & -0.33 & $-0.38,-0.28$ & $<0.001^{\wedge}$ \\
\hline
\end{tabular}

TB tuberculosis, PTB pulmonary tuberculosis, EPTB extrapulmonary tuberculosis, HIV human immunodeficiency virus

${ }^{a}$ registered in web-based TB information management system

blogarithmic transformation of delay variable done as it was not normally distributed, model building was done by stepwise (forward method) method and the final model has been presented; age group, gender, occupation, treatment category and registration at referral hospital, though considered, were excluded by the model (likelihood ratio test)

$F$ stat for model $=26.8$; Probability $>F=<0.001$

' of 7284, twenty two patient records were excluded because of missing

information on dates to calculate delay

${ }^{\wedge}$ significant $p$-value $<0.05$

and almost immediately after visiting a health facility in China [31] as other general populations [32].

We speculate that this was due to four important reasons. Firstly, most destinations of migration were urban areas and had obviously better universal health coverage [6, 7]. We did not adjust for this factor (urban/rural) in our analysis. Secondly, it might be related to the annual health examination (including TB) especially among people working at industries or companies where people migrated and worked, having high chance of early diagnosis and treatment [33, 34]. Thirdly, patients probably benefitted from the publicpublic mix collaboration among different public health providers and TB designated facilities which was implemented after severe acute respiratory syndrome epidemic [35]. Fourth, there were very few private practitioners in China, most health providers were public hospitals. According to national law and regulation of infectious diseases control, all health facilities must report TB cases within $24 \mathrm{~h}$ after diagnosis and refer them to programme BMUs since 2004 [36, 37]. BMUs must trace these referred patients with TB and get them involved in programme management [22]. These mandatory requirements possibly shortened the
Table 6 Linear regression for independent predictors of health system treatment initiation delay before registration among transferred out migrant patients with $\mathrm{TB}^{\mathrm{a}}$, China, $2014-15^{\mathrm{b}}(N=7262)^{\mathrm{c}}$

\begin{tabular}{|c|c|c|c|}
\hline Variable & B coefficient & $95 \% \mathrm{Cl}$ & $P$ value \\
\hline \multicolumn{4}{|l|}{ Occupation } \\
\hline Studying & 0.01 & $-0.01,0.04$ & 0.370 \\
\hline Farmers and herdsmen & -0.02 & $-0.04,-0.004$ & $0.013^{\wedge}$ \\
\hline Semi-skilled employee & -0.003 & $-0.05,0.05$ & 0.899 \\
\hline Salaried employee & -0.03 & $-0.05,-0.01$ & $0.002^{\wedge}$ \\
\hline Non-salaried employee & 0.001 & $-0.03,0.03$ & 0.970 \\
\hline Unemployed & Ref & Ref & Ref \\
\hline Others & -0.01 & $-0.04,0.02$ & 0.523 \\
\hline \multicolumn{4}{|l|}{ Permanent residence } \\
\hline Within prefecture & Ref & Ref & Ref \\
\hline Within province & -0.02 & $-0.04,0.01$ & 0.134 \\
\hline Out of province & -0.03 & $-.0 .05,-0.02$ & $<0.001^{\wedge}$ \\
\hline \multicolumn{4}{|l|}{ Classification } \\
\hline PTB smear positive & 0.02 & $-0.002,0.05$ & $0.071^{\wedge}$ \\
\hline PTB smear negative & -0.01 & $-0.04,0.01$ & 0.391 \\
\hline PTB smear status unknown & 0.25 & $0.16,0.35$ & $<0.001^{\wedge}$ \\
\hline Pleurisy & Ref & Ref & Ref \\
\hline EPTB & -0.03 & $-0.33,0.27$ & 0.830 \\
\hline \multicolumn{4}{|l|}{ Category } \\
\hline New & -0.04 & $-0.07,-0.01$ & $0.014^{\wedge}$ \\
\hline Retreated & Ref & Ref & Ref \\
\hline \multicolumn{4}{|l|}{ HIV } \\
\hline Positive & -0.07 & $-0.24,0.10$ & 0.425 \\
\hline Negative & Ref & Ref & Ref \\
\hline Unknown & -0.34 & $-0.05,-0.02$ & $<0.001^{\wedge}$ \\
\hline
\end{tabular}

TB tuberculosis, PTB pulmonary tuberculosis, EPTB extrapulmonary tuberculosis, HIV human immunodeficiency virus

${ }^{\text {a }}$ registered in web-based TB information management system

${ }^{b}$ logarithmic transformation of delay variable done as it was not normally distributed, model building was done by stepwise (forward method) method and the final model has been presented; age group, gender and registration at referral hospital, though considered, were excluded by the model (likelihood ratio test)

$F$ stat for model $=10.6 ;$ Probability $>F=<0.001$

cof 7284, twenty two patient records were excluded because of missing information on dates to calculate delay

$\wedge$ significant $p$-value $<0.05$

health system diagnosis delay compared to historical studies before in China [38, 39]. On the other hand, providing free anti-tuberculosis drugs by programme BMUs reduced the health system treatment delay [22], which was also relatively shorter than most other low-middle income countries [4, 13, 40], while similar as in South Africa [41].

\section{Predictors of patient delay, diagnosis and total delay} Independent predictors for long patient delay, diagnosis delay and total delay were the same (elderly age group, 
Table 7 Linear regression for independent predictors of diagnosis delay before registration among transferred out migrant patients with $\mathrm{TB}^{\mathrm{a}}$, China, 2014-15 ${ }^{\mathrm{b}}(\mathrm{N}=7262)^{\mathrm{c}}$

\begin{tabular}{llll}
\hline Variable & B coefficient & $95 \% \mathrm{Cl}$ & $P$ value \\
\hline Age group (in years) & & & \\
$<15$ & -0.26 & $-0.69,0.17$ & 0.240 \\
$15-44$ & -0.10 & $-0.19,-0.01$ & $0.037^{\wedge}$ \\
$45-64$ & 0.03 & $-0.07,0.13$ & 0.560 \\
$>=65$ & Ref & Ref & Ref \\
Gender & & & \\
Male & Ref & Ref & Ref \\
Female & 0.11 & $0.05,0.18$ & $<0.001^{\wedge}$ \\
Classification & & & \\
PTB smear positive & 0.21 & $0.08,0.33$ & $0.001^{\wedge}$ \\
PTB smear negative & -0.01 & $-0.13,0.10$ & 0.814 \\
PTB smear status unknown & 0.44 & $0.01,0.87$ & $0.047^{\wedge}$ \\
Pleurisy & Ref & Ref & Ref \\
EPTB & 0.53 & $-0.88,1.95$ & 0.462 \\
Registered at referral hospital & & & \\
Yes & Ref & Ref & Ref \\
No & -0.06 & $-0.13,-0.003$ & $0.037^{\wedge}$ \\
\hline
\end{tabular}

TB tuberculosis, PTB pulmonary tuberculosis, EPTB extrapulmonary tuberculosis ${ }^{a}$ registered in web-based TB information management system

${ }^{b}$ logarithmic transformation of delay variable done as it was not normally distributed, model building was done by stepwise (forward method) method and the final model has been presented; occupation, permanent residence, treatment category and HIV status, though considered, were excluded by the model (likelihood ratio test)

$F$ stat for model $=9.28$; Probability $>F=<0.001$

cof 7284 , twenty two patient records were excluded because of missing information on dates to calculate delay

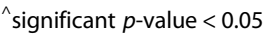

female gender, patients registered in referral hospitals and patients with pulmonary sputum smear positive TB). It may be because patient delay contributed to most of the diagnosis and total delay.

Female migrant TB patients being associated with delays has also been reported elsewhere/before in China, India, and Asia [11, 12, 29]. Females might prefer selftreatment using home remedies to treat their TB like symptoms at home which might lead to delay in seeking care. Compared with pleurisy, patients with sputum smear positive were associated with delay. It might be that TB symptoms were relatively more severe among pleurisy patients [42]. This study also found that patients with 1544 years of age were less likely to have delays than older age group. This finding contradicts with the finding from India where patients $<45$ years of age were at higher risk for delay [43]. Patients who were registered by referral hospitals had longer delay when compared to programme BMUs. This could be explained by the fact that patients visited referral hospital after visiting other public facilities or after becoming sick.
Table 8 Linear regression for independent predictors of total delay before registration among transferred out migrant patients with $\mathrm{TB}^{\mathrm{a}}$, China, $2014-15^{\mathrm{b}}(N=7262)^{\mathrm{c}}$

\begin{tabular}{|c|c|c|c|}
\hline Variable & B coefficient & $95 \% \mathrm{Cl}$ & $P$ value \\
\hline \multicolumn{4}{|l|}{ Age group (in years) } \\
\hline$<15$ & -0.23 & $-0.65,0.20$ & 0.300 \\
\hline $15-44$ & -0.11 & $-0.20,-0.01$ & $0.024^{\wedge}$ \\
\hline $45-64$ & 0.03 & $-0.07,0.12$ & 0.605 \\
\hline$>=65$ & Ref & Ref & Ref \\
\hline \multicolumn{4}{|l|}{ Gender } \\
\hline Male & Ref & Ref & Ref \\
\hline Female & 0.11 & $0.05,0.18$ & $<0.001^{\wedge}$ \\
\hline \multicolumn{4}{|l|}{ Classification } \\
\hline PTB smear positive & 0.21 & $0.09,0.33$ & $0.001^{\wedge}$ \\
\hline PTB smear negative & -0.02 & $-0.14,0.10$ & 0.740 \\
\hline PTB smear status unknown & 0.53 & $0.10,0.96$ & $0.015^{\wedge}$ \\
\hline Pleurisy & ref & & \\
\hline EPTB & 0.54 & $0.85,1.93$ & 0.445 \\
\hline \multicolumn{4}{|l|}{ HIV } \\
\hline Positive & 0.61 & $-0.19,1.41$ & 0.134 \\
\hline Negative & Ref & Ref & Ref \\
\hline Unknown & -0.04 & $-0.10,0.02$ & 0.166 \\
\hline \multicolumn{4}{|l|}{ Registered at referral hospital } \\
\hline Yes & Ref & Ref & Ref \\
\hline No & -0.07 & $-0.13,-0.003$ & $0.038^{\wedge}$ \\
\hline
\end{tabular}

TB tuberculosis, PTB pulmonary tuberculosis, EPTB extrapulmonary tuberculosis, HIV human immunodeficiency virus

${ }^{a}$ registered in web-based TB information management system blogarithmic transformation of delay variable done as it was not normally distributed, model building was done by stepwise (forward method) method and the final model has been presented; occupation, permanent residence and treatment category, though considered, were excluded by the model (likelihood ratio test)

$F$ stat for model $=9.1$; Probability $>F=<0.001$

cof 7284, twenty two patient records were excluded because of missing information on dates to calculate delay

^significant $p$-value $<0.05$

\section{Predictors of health system delay}

Among migrant patients having permanent residence out of province, health system diagnosis delay was significantly higher while treatment initiation delay was significantly lower when compared to migrant patients having permanent residence within the prefecture. This was intriguing. The former needs to be addressed by the programme.

Contrarily, sputum smear positive PTB in the model of patient delay was an independent predictor for longer delay but in the model of health system diagnosis delay was an independent predictor for shorter delay. It may that smear positive PTB patients were easier to be diagnosed for bacteriological evidence, more likely to be infectious and NTP paid more attention to them than TB pleurisy patients. 
Previously treated patients had significantly higher treatment initiation delay which could be because of their previous bad experience of TB treatment, similar as reported elsewhere/before in India and China [13, 32]. Patient registered in referral hospital had significant higher treatment initiation delay after diagnosis due to procedural and interest reasons [44].

\section{Policy implications}

Though programme is doing well in shortening patient delays when compared to other countries, however, these can be further reduced if we want to move towards ending TB [45]. Migrant populations were less likely to be covered by social insurance, which not only caused delays due to their health seeking behaviors, but also led to high risk of transfer-out during the treatment (programme records). In order to change this situation, firstly migrant population needs to be treated equally under universal health coverage. Providing subsidies to migrant patients with TB could effectively encourage them visit doctors and reduce the patient delay, and ensure them stay in the diagnosed place to complete treatment $[46,47]$.

Lack of knowledge of TB symptoms, poor policy awareness and stigma were the most common risk factors identified previously with patient delay which could be even more among elderly and women migrants [12, 28-30]. The programme should focus on health promotion activities among elderly and women migrants who are probably family numbers of migrant workers and have less access to work place interventions. In addition, active case finding in migrant population could also reduce the patient delay [9].

Around two-thirds of patients in our study were registered in referral hospitals and had delayed diagnosis and treatment initiation. China must consider ameliorating the implementation of NTP policies in referral hospitals. Referral hospitals have less motivation to involve patients in programme and complete the full course treatment because of free drugs and standardized follow-up examinations [44]. It is imperative to build efficient coordination mechanisms between referral hospitals and national programme. More studies need to be implemented to explore the mechanisms and activities which could eventually help reducing patient level, diagnosis and treatment initiation delay.

\section{Strengths and limitations}

Few countries collect sufficiently disaggregated data on the health of migrants $[5,48]$ and this is a major strength of the study. Sustainable development goal number 17 includes two targets and associated indicators under the subheading of 'data, monitoring and accountability' which include mechanism to generate disaggregate data for specific subpopulations. Migrants are one such subpopulation. [1,49] Second, the findings are representative of the situation in China as the study involved a national cohort of patients over 2 years without sampling. Third, there were minimal missing values, despite being a record review study.

This study could not include some important factors which influence delay, such as knowledge and attitude, type of health care provider visited, information on self-medications, smoking and alcohol use, family income, urban/rural residence and nutritional status. This information is not routinely collected by the TBIMS. The cross-sectional nature of data limited causal inference. Some speculations were discussed based on our experience of working within programme; future qualitative research is needed to further clarify these speculations. Finally, there are concerns regarding applying and interpreting the results of hypothesis testing in a log-transformed data on non-log-transformed data [50].

\section{Conclusion}

Limitations notwithstanding, our findings indicate that delay before registration among migrant $\mathrm{TB}$ patients was lower than general population in China and globally. Patient delay contributed to the total delay. Elderly patients, females, those with sputum smear positive pulmonary TB and those registered in referral hospital had higher patient, diagnosis and total delay. Patients with permanent residence out of province had significantly higher delay in diagnosis after visiting a health facility. Patients registered in referral hospital also had significantly higher treatment initiation delay after diagnosis. If China has to attain the targets of ending TB by 2035 [45], these factors including improved coordination between referral hospitals and programme have to be addressed urgently.

\section{Additional file}

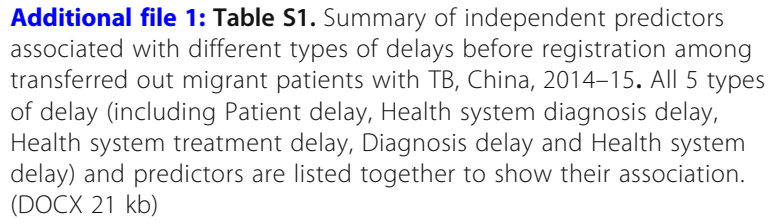

Additional file 1: Table S1. Summary of independent predictors associated with different types of delays before registration among transferred out migrant patients with TB, China, 2014-15. All 5 types of delay (including Patient delay, Health system diagnosis delay, Health system treatment delay, Diagnosis delay and Health system delay) and predictors are listed together to show their association. (DOCX $21 \mathrm{~kb}$ )

\section{Abbreviations}

BMU: Basic management unit; IQR: Interquartile range; NCTB: National Center for Tuberculosis Control and Prevention; PTB: Pulmonary TB; TB: Tuberculosis; TBIMS: TB information management system; WHO: World Health Organization

\section{Acknowledgements}

This research was conducted through the Structured Operational Research and Training Initiative (SORT IT), a global partnership led by the Special

Program for Research and Training in Tropical Diseases at the World Health Organization (WHO/TDR). The model is based on a course developed jointly by the International Union Against Tuberculosis and Lung Disease (The Union) and 
Medécins sans Frontières (MSF/Doctors Without Borders). The specific SORT IT program which resulted in this publication was jointly developed and implemented by: The Union South-East Asia Office, New Delhi, India; the Center for Operational Research, The Union, Paris, France; The Union, Mandalay, Myanmar; MSF Luxembourg Operational Research (LuxOR); MSF Operational Center Brussels (MSF OCB); Institute of Medicine, University of Chester, UK; and Department of Medical Research, Ministry of Health and Sports, The Republic of The Union of Myanmar.

\section{Funding}

The author(s) received no specific funding for this work. The training programme were funded by the Department for International Development (DFID), UK. The funders had no role in study design, data collection and analysis, decision to publish, or preparation of the manuscript. The open access fee for the publication is funded by WHO West Pacific Office through Technical Services project. The project number is WPDCC1408653.

\section{Availability of data and materials}

The authors confirm that all data underlying the findings are fully available without restriction and the clean dataset is available upon request to authors.

\section{Disclaimer}

The contents of this paper do not necessarily reflect the views of the National Center for Tuberculosis Control and Prevention, China CDC, China; The Union; or Department of Medical Research Ministry of Health and Sports, The Republic of The Union of Myanmar.

\section{Authors' contributions}

$T L, H D S, K T S$ were involved in conception and study design; TL was involved in data extraction; TL, HDS, KTS, XD, LW and $H Z$ were involved in analysis and interpretation of data; $X D, L W$ and $H Z$ were involved in overall supervision; HDS and KTS are the mentors, XD is the senior author. All authors read and approved the final manuscript.

\section{Ethics approval and consent to participate}

Ethics approval was obtained from the Ethics Advisory Group of The Union, Paris, France (EAG No: 30/17) and Ethics Committee of Chinese Center for Disease Control and Prevention (Number: 201704). Permission for the study was sought from National Center for Tuberculosis Control and Prevention of China (NCTB) before initiating the study. As this was a study involving record review, waiver of written informed consent was sought and waived off by the ethics committees.

\section{Consent for publication}

Not applicable.

\section{Competing interests}

The authors declare that they have no competing interests.

\section{Publisher's Note}

Springer Nature remains neutral with regard to jurisdictional claims in published maps and institutional affiliations.

\section{Author details \\ ${ }^{1}$ National Center for Tuberculosis Control and Prevention, China CDC, No. 155 Changbai Road, Changping District, Beijing 102206, China. ${ }^{2}$ International Union Against Tuberculosis and Lung Disease (The Union), South-East Asian Office, Delhi, India. ${ }^{3}$ Department of Medical Research (PyinOoLwin Branch), Ministry of Health and Sports, PyinOoLwin, Myanmar.}

\section{Received: 23 January 2018 Accepted: 28 September 2018}

Published online: 19 October 2018

\section{References}

1. World Health Organization (WHO). Global tuberculosis report 2017. Geneva: WHO/HTM/TB/2017.23; 2017.

2. World Health Organization (WHO). Global strategy and targets for tuberculosis prevention, care and control after 2015. Geneva:WHA65/2012/REC/3; 2013.

3. Gebreegziabher SB, Bjune GA, Yimer SA. Total delay is associated with unfavorable treatment outcome among pulmonary tuberculosis patients in west Gojjam zone, Northwest Ethiopia: a prospective cohort study. PLoS One. 2016;11:1-16.

4. Virenfeldt J, Rudolf F, Camara C, Furtado A, Gomes V, Aaby P, et al. Treatment delay affects clinical severity of tuberculosis: a longitudinal cohort study. BMJ Open. 2014;4:e004818.

5. World Health Organization Regional Office for the Western Pacific. Tuberculosis Control in Migrant Populations: Guiding Principles and Proposed Actions. Manila: WHO Press; 2016.

6. China Statistics Press. China statistical yearbook 2015. 2016. Available from: http://www.stats.gov.cn/tjsj/ndsj/2015/indexch.htm. [cited 15 Nov 2017].

7. National Bureau of Statistics of the People's Republic of China. Monitoring report of rural-to-urban migrant workers in 2015. 2016. Available from: http://www.stats.gov.cn/tjsj/zxfb/201604/t20160428_ 1349713.html. [cited 15 Nov 2017].

8. Sun Y-X, Zhu L, Lu Z-H, Jia Z-W. Notification rate of tuberculosis among migrants in China 2005-2014: a systematic review and meta-analysis. Chin Med J. 2016;129:1856.

9. Ndeikoundam Ngangro N, Chauvin P, Halley des Fontaines V. Determinants of tuberculosis diagnosis delay in limited resources countries. Rev Epidemiol Sante Publique. 2012;60:47-57.

10. Heuvelings CC, de Vries SG, Greve PF, Visser BJ, Bélard S, Janssen S, et al. Effectiveness of interventions for diagnosis and treatment of tuberculosis in hard-to-reach populations in countries of low and medium tuberculosis incidence: a systematic review. Lancet Infect Dis. 2017;17:e144-58.

11. Cai J, Wang X, Ma A, Wang Q, Han X, Li Y. Factors associated with patient and provider delays for tuberculosis diagnosis and treatment in Asia: a systematic review and meta-analysis. PLoS One. 2015;10:e0120088.

12. Li Y, Ehiri J, Tang S, Li D, Bian Y, Lin H, et al. Factors associated with patient, and diagnostic delays in Chinese TB patients: a systematic review and metaanalysis. BMC Med. 2013;11:156.

13. Samal J. Health seeking behaviour among tuberculosis patients in India: a systematic review. J Clin Diagnostic Res. 2016;10:LE01-6.

14. Peng Y, Chang W, Zhou H, Hu H, Liang W. Factors associated with healthseeking behavior among migrant workers in Beijing, China. BMC Health Serv Res. 2010;10:69.

15. Liang Q-F, Pang Y, Chen Q-Y, Lin S-F, Lin J, Zhao Y, et al. Genetic profile of tuberculosis among the migrant population in Fujian Province, China. Int J Tuberc Lung Dis. 2013;17:655-61.

16. Li X, Jiang S, Li X, Mei J, Zhong Q, Xu W, et al. Predictors on delay of initial health-seeking in new pulmonary tuberculosis cases among migrants population in East China. PLoS One. 2012;7:e31995.

17. Tobe RG, Xu L, Song P, Huang Y. The rural-to-urban migrant population in China: gloomy prospects for tuberculosis control. Biosci Trends. 2011; 5:226-30.

18. Abarca Tomás B, Pell C, Bueno Cavanillas A, Guillén Solvas J, Pool R, Roura M. Tuberculosis in migrant populations. A systematic review of the qualitative literature. PLoS One. 2013:8:e82440.

19. Zhang Y, Kan X, Han L. Analysis on delayed-detection situation of new smear positve cases with pulmonary tuberculosis in Anhui. Anhui J Prev Med. 2011;17:329-329,356.

20. Gneg H, Zhou C, Liu Z, Xu L, Tao W, Li H, et al. Delay in seeking medical consultation among pulmonary tuberculosis patients in migrants. Chin $J$ Public Heal. 2010;26:977-8.

21. He S, Guo J. Factors associated with diagnostic delay of 142 PTB. China Mod Med. 2009;16:21-2

22. Ministry of Health. Guidelines for implementing the National Tuberculosis Control Program in China (2008). Beijing: Press of Chinese Peking Union Medical College; 2009.

23. Lin Y, Enarson DA, Chiang C-Y, Rusen ID, Qiu L-X, Kan X-H, et al. Patient delay in the diagnosis and treatment of tuberculosis in China: findings of case detection projects. Public Heal Action. 2015;5:65-9.

24. Shu W, Chen W, Zhu S, Hou Y, Mei J, Bai L, et al. Factors causing delay of access to tuberculosis diagnosis among new, active tuberculosis patients: a prospective cohort study. Asia-Pac J Public Heal. 2014:26:33-41.

25. Zhou C, Chu J, Geng H, Wang X, Xu L. Pulmonary tuberculosis among migrants in Shandong, China: factors associated with treatment delay. BMJ Open. 2014;4:e005805.

26. Chen W, Zhang Q, Renzaho AMN, Zhou F, Zhang H, Ling L. Social health insurance coverage and financial protection among rural-to-urban internal migrants in China: evidence from a nationally representative cross-sectional study. BMJ Glob Heal. 2017;2:e000477. 
27. Huang F, Cheng S, Du X, Chen W, Scano F, Falzon D, et al. Electronic recording and reporting system for tuberculosis in China: experience and opportunities. J Am Med Inform Assoc. 2014;21:938-41.

28. Storla DG, Yimer S, Bjune GA. A systematic review of delay in the diagnosis and treatment of tuberculosis. BMC Public Health. 2008;8:15.

29. Sreeramareddy CT, Qin ZZ, Satyanarayana S, Subbaraman R, Pai M, Zhen Qin $Z$, et al. Delays in diagnosis and treatment of pulmonary tuberculosis in India: a systematic review. Int J Tuberc Lung Dis. 2014;18:255-66.

30. Sreeramareddy CT, Panduru KV, Menten J, Van den Ende J. Time delays in diagnosis of pulmonary tuberculosis: a systematic review of literature. BMC Infect Dis. 2009:9:91.

31. Deng $H$, Zheng $Y$, Zhang $Y, X u B$. Study on factors causing the delay of access to tuberculosis diagnosis and its influencing factors in migrating tuberculosis patients in Putuo district. Shanghai Chinese J Epidemiol. 2006:27:311-5.

32. He Y, Han C, Chang K-F, Wang M-S, Huang T-R. Total delay in treatment among tuberculous meningitis patients in China: a retrospective cohort study. BMC Infect Dis. 2017;17:341.

33. Yue W, Zhao H, Cao S, Guo J, Zhang G, Wang G. Analysis of results of pulmonary tuberculosis screening in health checkup among food and public places employees in 2009-2014 in Pingdingshan. Chinese J Pest Control. 2016:32:148-51.

34. Lin $\mathrm{C}, \mathrm{Xu}$ J, Li J, Wang F, Zhang $\mathrm{H}$, Wang $\mathrm{H}$, et al. Screening for tuberculosis in health examination participants and clinical study on the spontaneous occurence of tuberculosis in the populations with strong positive reaction to tuberculin skin test in ten years. Chin J Heal Manag. 2015;9:69-72.

35. Wang L, Liu X, Huang F, Hennig C, Uplekar M, Jiang S. Engaging hospitals to meet tuberculosis control targets in China: using the internet as a tool to put policy into practice. Bull World Health Organ. 2010;88:937-42.

36. Bureau of Diseases Control and Prevention of NHFPC. Standards for information report of infectious diseases(2015). 2015. Available from: http:// www.gov.cn/xinwen/2015-11/11/content 2964135.htm. [cited 7 Jan 2018]

37. Standing Committee of the National People's Congress. Law of the People's Republic of China on the Prevention and Treatment of Infectious Diseases. 2004. Available from: http://www.gov.cn/gongbao/content/2004/content_ 62975.htm. [cited 7 Jan 2018]

38. Chen Q, Zheng J, Wu N. Analysis on the correlated factors of delay in tuberculosis diagnosis and treatment in Fujian province. Chin J Antituberc. 2005;27:374-9.

39. Bai $L$, Xiao $S$. Factors associated with diagnostic delay for patients with smear-positive pulmonary tuberculosis in rural Hunan, China. Chin J Tuberc Respir Dis. 2004;27:617-20.

40. Adenager GS, Alemseged F, Asefa H, Gebremedhin AT. Factors associated with treatment delay among pulmonary tuberculosis patients in public and private health facilities in Addis Ababa, Ethiopia. Tuberc Res Treat. Hindawi Publishing Corporation. 2017;2017:1-9.

41. Van Wyk SS, Enarson DA, Beyers N, Lombard C, Hesseling AC. Consulting private health care providers aggravates treatment delay in urban south African tuberculosis patients. Int J Tuberc Lung Dis. 2011:15:1069-76.

42. Qiu L, Teeter LD, Liu Z, Ma X, Musser JM, Graviss EA. Diagnostic associations between pleural and pulmonary tuberculosis. J Inf Secur. 2006;53:377-86

43. Kulkarni PY, Kulkarni AD, Akarte SV, Bhawalkar JS. Treatment seeking behavior and related delays by pulmonary tuberculosis patients in E-ward of Mumbai municipal corporation, India. Int J Med Public Heal. 2013;3:286-92.

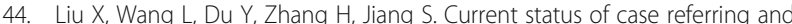
treatment of tuberculosis impatients in four tuberculosis specialist hospitals in China. Chin J Antituberc. 2010;32:775-8.

45. World Health Organization (WHO). Implementing the end TB strategy: the essentials. Geneva: WHO/HTM/TB/2015.31; 2015.

46. Zhao Q, Wang L, Tao T, Xu B. Impacts of the "transport subsidy initiative on poor TB patients" in rural China: a patient-cohort based longitudinal study in rural China. PLoS One. 2013:8:e82503.

47. Lu H, Yan F, Wang W, Wu L, Ma W, Chen J, et al. Do transportation subsidies and living allowances improve tuberculosis control outcomes among internal migrants in urban Shanghai, China? West Pacific Surveill Response J. 2013:4:19-24.
48. World Health Organization (WHO). Health of migrants migration flows and the globalized world. Geneva: EB122/11; 2007.

49. World Health Organization. Health in 2015: from MDGs, Millennium Development Goals to SDGs, Sustainable Development Goals. Geneva: WHO Press; 2015

50. Feng C, Wang H, Lu N, Chen T, He H, Lu Y, et al. Log-transformation and its implications for data analysis. Shanghai Arch Psychiatry. 2014;26:105-9.
Ready to submit your research? Choose BMC and benefit from:

- fast, convenient online submission

- thorough peer review by experienced researchers in your field

- rapid publication on acceptance

- support for research data, including large and complex data types

- gold Open Access which fosters wider collaboration and increased citations

- maximum visibility for your research: over $100 \mathrm{M}$ website views per year

At BMC, research is always in progress.

Learn more biomedcentral.com/submissions 